\title{
ON THE USE OF POINT CONTACT MODELS FOR COLLAPSE MECHANISM AND DYNAMIC ANALYSIS OF MASONRY STRUCTURES
}

\author{
Francesco Portioli ${ }^{1}$, Lucrezia Cascini ${ }^{2}$, and Raffaele Landolfo ${ }^{2}$ \\ ${ }^{1}$ University of Naples Federico II, Department of Structures for Engineering and Architecture, Via \\ Forno Vecchio 36, 80134 Naples \\ e-mail: fportiol@unina.it \\ ${ }^{2}$ University of Naples Federico II, Department of Structures for Engineering and Architecture, Via \\ Forno Vecchio 36, 80134 Naples \\ \{lucrezia.cascini, landolfo\}@unina.it
}

Keywords: Masonry block structures; Rigid blocks; Limit analysis; Non-smooth contact dynamics; Mathematical programming; Collapse mechanism analysis; Rocking behavior.

\begin{abstract}
In this paper, we discuss the main issues concerning the use of point-based models to represent contact interactions in computational limit analysis and non-smooth contact $d y$ namics of masonry structures. To investigate the response of masonry structures, numerical models composed of rigid blocks interacting at contact points located at the vertexes of the interfaces are used. A no-tension behavior with finite friction and infinite compressive strength is assumed at contact interfaces. The limit analysis problem and the contact dynamic problem are formulated in terms of equilibrium equations, which relate external and contact forces, kinematic equations, which ensure compatibility between contact displacement rates and block degrees of freedom, and additional relationships to model the behavior of contacts. Mathematical programming is used to solve the optimization problems arising from both limit analysis and the dynamic of the block assemblages. The accuracy and computational efficiency of the implemented formulations are discussed with reference to case studies selected from the literature.
\end{abstract}




\section{INTRODUCTION}

Recent studies on rigid block modelling of masonry structures revealed that the use of pointcontact models leads to simple and efficient formulations both in the case of collapse mechanism analysis of buildings subjected to lateral loads and settlement induced effects, as well as in the case of dynamic analysis under ground motion excitation [1-5].

When a point contact model is adopted to represent interactions, it is assumed that the blocks interact via a number of contact points, which are usually located at the corners of the interface. In this case, the static variables associated to contact interactions are simply related to normal and shear forces at a contact point. This modelling approach represents an alternative method to the classic surface contact formulation, where stress resultants acting at a single point are considered to represent contact interaction, thus including bending and eventually torsion moments (the last ones in the case of $3 \mathrm{D}$ formulations) in addition to normal and shear forces [1, $3,6]$.

In the case of the point contact model and when a no-tension, associative frictional behavior for displacement rates is assumed, the equations governing the limit equilibrium analysis and the contact dynamic problem are simplified considerably and can be cast as simple mathematical programming problems, for which very efficient solution algorithms exist $[3,7]$.

In this paper, we present the formulations previously developed in $[3,7]$ according to a unified approach, in order to point out similarities and differences between the limit equilibrium analysis and contact dynamics problems of dry-jointed masonry structures.

The manuscript is organized as follows. In Sections 2, the static and kinematic variables as well as the relationships governing the behavior of the rigid block model are presented. The formulation of the mathematical programming problems that are used to solve limit analysis and the contact dynamic problems are reported in Section 3. In Section 4, a numerical case study taken from the literature and consisting into a set of block panels subjected to lateral loads is considered for validation and to compare the limit equilibrium and the dynamic formulation.

\section{THE RIGID BLOCK MODEL FOR COLLAPSE MECHANISM AND DYNAMIC ANALYSIS}

The masonry panel is discretized into a multi-body assemblage made of rectangular rigid blocks $i$ interacting at potential contact points $k$ located at the vertexes of the interface $j$ (Fig. 1). Although the modelling approach proposed can be extended to irregular textures and block shapes, in this study we assume a regular stretcher bonded (or 'running bond') texture, with the blocks staggered in each successive course by half a block.

A no-tension and associative frictional behavior with infinite compressive strength is assumed at contact interfaces.

The contact variables are the internal forces acting at each contact point $k$, which are located at a vertex of interface $j$ of block $i$ (Fig. 2a). These variables are collected in vector $c$ and include the shear force component $t_{k}$ and the normal force $n_{k}$ along the local coordinate axes.

External loads applied to the centroid of rigid block $i$ are collected in vector of external forces $f$ (Fig. 2a).

The kinematic variables associated in a virtual work sense to the contact forces are the relative displacement rates at the contact points, namely the tangential and normal displacement rates $\Delta u_{t k}$ and $\Delta u_{n k}$ (Fig. 2b), which are collected in the vector $\boldsymbol{\Delta} \boldsymbol{u}$. The positions $x_{i}, y_{i}$ and $\omega_{i}$ related to the degrees of freedom of each block $i$ are collected in the vector $\boldsymbol{x}$. 


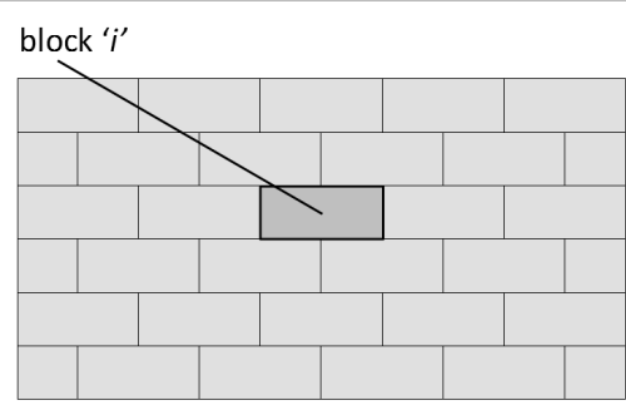

a)

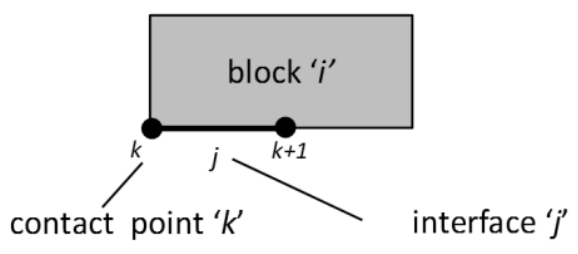

b)

Figure 1: a) Rigid block assemblage; b) Rigid block $i$, interface $j$ and contact point $k$.
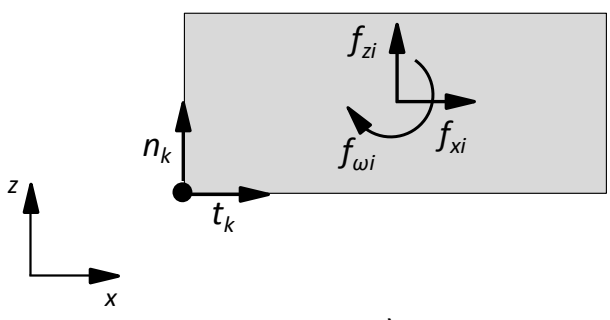

a)

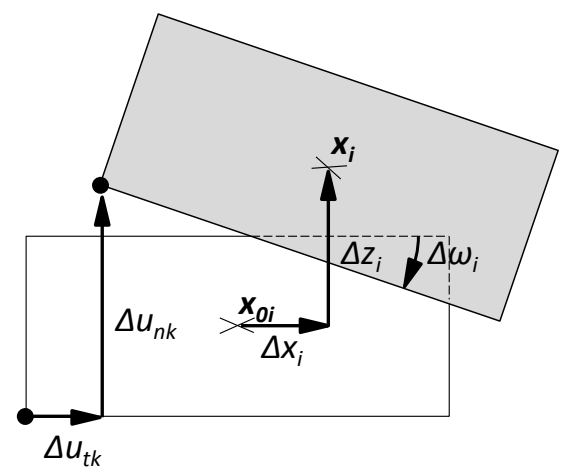

b)

Figure 2: a) Contact forces and b) kinematic variables at block centroid $i$, and contact point $k$.

The dynamic model is formulated following the approach proposed in [8-11] for granular materials, though now detailed for two-dimensional assemblages of rectangular blocks [7].

The equations of motions are discretized with respect to time using the $\theta$-method and assuming:

$$
\dot{x}(t)=\frac{1}{\theta}\left[\frac{\Delta x}{\Delta t}-(1-\theta) \dot{x}_{0}\right]
$$

where $\boldsymbol{\Delta} \boldsymbol{x}=\boldsymbol{x}-\boldsymbol{x}_{\mathbf{0}}$ is the displacement vector, $\boldsymbol{x}_{\mathbf{0}}$ and $\dot{\boldsymbol{x}}_{\mathbf{0}}$ are the known position and velocity at time $t_{0}$ and $\theta \geq 0.5$.

On the basis of the incremental expression (1), the equations of motion of the rigid block assemblage interacting at potential contact points can be posed as follows:

$$
\bar{M} \Delta x+A_{0} c=\bar{f}_{0}
$$

where:

$\boldsymbol{A}_{\mathbf{0}}$ is the equilibrium matrix corresponding to contact forces; 
$\overline{\boldsymbol{M}}=\frac{1}{\theta \Delta t^{2}} \boldsymbol{M}$, being $\boldsymbol{M}$ the mass matrix collecting the mass $m_{i}$ and the mass moment of inertia $J_{i}$ of each block;

$\overline{\boldsymbol{f}}_{\mathbf{0}}=\boldsymbol{f}+\overline{\boldsymbol{M}} \dot{\boldsymbol{x}}_{0} \Delta t$

Contact conditions are expressed imposing that the normal component of the relative displacement at contact point $k$ is lesser than or equal to the initial gap, as follows:

$$
\widehat{\boldsymbol{n}}_{\mathbf{0} \boldsymbol{k}}^{\mathbf{T}} \boldsymbol{\Delta} \boldsymbol{u}_{\boldsymbol{k}} \leq \mathrm{g}_{0 k}
$$

where $\widehat{\boldsymbol{n}}_{\mathbf{0} \boldsymbol{k}}$ is the initial normal associated with the surface $j$ and $g_{0 k}$ is the initial gap.

For contact interactions, a complementarity condition is included as follows:

$$
n_{k}\left[\widehat{\boldsymbol{n}}_{\mathbf{0} \boldsymbol{k}}^{\mathbf{T}} \boldsymbol{\Delta} \boldsymbol{u}_{\boldsymbol{k}}-\mathrm{g}_{0 k}\right]=0
$$

Considering that $n_{k} \geq 0$, this condition ensures that contact forces occur only if the gap is closed otherwise are zero.

Eqs. (6), (7) express the so-called Signorini unilateral contact conditions at a contact point $k$ in terms of displacement rates.

The behaviour at contact interfaces undergoing sliding failure is governed by failure conditions which are expressed according to the Coulomb friction law.

In vector notation, the limit conditions for sliding failure can be written as:

$$
\pm \boldsymbol{t} \leq \mu \boldsymbol{n}
$$

where $\mu$ is the friction coefficient.

\section{FORMULATION OF THE MATHEMATICAL PROGRAMMING PROBLEMS}

Under the assumption of associative flow rule for displacement rates, the equilibrium equations, kinematic conditions and sliding friction conditions are equivalent to the following forcebased problem [7-11]:

$$
\begin{array}{ll}
\max & -\frac{1}{2} \boldsymbol{r}^{\mathbf{T}} \overline{\boldsymbol{M}}^{-\mathbf{1}} \boldsymbol{r}-\boldsymbol{g}_{\mathbf{0}}^{\mathbf{T}} \boldsymbol{c} \\
\text { s.t. } & \boldsymbol{r}+\boldsymbol{A}_{\mathbf{0}} \boldsymbol{c}=\overline{\boldsymbol{f}}_{\mathbf{0}} \\
& \pm \boldsymbol{t}-\mu \boldsymbol{n} \leq \mathbf{0}, \quad \boldsymbol{n} \geq \mathbf{0}
\end{array}
$$

where $\boldsymbol{r}$ is the vector of dynamic forces associated with the degrees of freedom of the blocks.

If we now express external forces as the sum of dead and live loads multiplied by the collapse load factor, the formulation of the limit equilibrium problem can be posed as follows : 


$$
\begin{array}{cl}
\max & \alpha \\
\text { s.t. } & \boldsymbol{A}_{\mathbf{0}} \boldsymbol{c}=\boldsymbol{f}_{\boldsymbol{D}}+\alpha \boldsymbol{f}_{\boldsymbol{L}} \\
& \pm \boldsymbol{t}-\mu \boldsymbol{n} \leq \mathbf{0}, \quad \boldsymbol{n} \geq \mathbf{0}
\end{array}
$$

It is interesting to note that, in the limit of dynamic forces and contact gaps tending to zero, the dynamic problem (9) is formally equivalent to the one presented in (10), provided that the vector external forces reported in (9) is properly decomposed in terms of dead and live loads as in (10).

The optimization problems (9) and (10) were actually implemented and solved by posing that the kinematic variables are derived as Lagrange multipliers associated with the various constraints, and implementing an incremental procedure to calculate and update positions of the blocks and contact gaps in the case of dynamic analysis. Further details on the assumptions behind the formulation and on the theoretical basis of the proposed formulations can be found in [7].

From an operative point of view, the proposed solution procedure was implemented in a computer code, DynoBlock_2D, which provides as outputs the time histories of contact forces and kinematic variables as well as the plots of the failure mechanisms at different time steps.

The mathematical programming problems associated to (9) and (10) were solved using the primal-dual interior-point solver in MOSEK [12].

The analyses were carried out using a PC containing a 3.3GHz Intel Xeon E3-1245 processor with 8 GB of RAM.

\section{APPLICATION TO IN-PLANE LOADED MASONRY WALLS}

In this section the block panels analyzed by Ferris and Tin-Loi [13] and subsequently investigated by Gilbert et al. [14] using different formulations for contact models and computational limit analysis are considered. The main goal of this analysis was to compare results obtained with the point contact formulation with those previously obtained within the framework of limit equilibrium analysis adopting a surface contact model [14].

The investigated wall panels are subject to vertical and in-plane horizontal loads. The size of a full block is $0.4 \times 0.175 \mathrm{~m}$, the friction coefficient is 0.65 and the unit weight is $1.0 \mathrm{kN} / \mathrm{m}^{3}$. To simulate the load cases of the limit analysis formulation, where each full block is subject to a horizontal live load expressed as a factor of the block weight, in the contact dynamic formulation the blocks are subject to a constant value of horizontal acceleration and to the gravity acceleration. The analysis is repeated increasing the horizontal acceleration by step of $0.001 \mathrm{~g}$ until rocking motion is initiated. Once the minimum value of acceleration is obtained, a constant acceleration is applied to the panel to analyze the evolution of the failure mechanism over time.

The sizes of the numerical models, expressed as the number of blocks $b$ and contact points $c$, are reported in Table 1, together with the numerical results in terms of accelerations and ultimate load factors obtained from limit equilibrium analysis, as well as CPU times. For contact dynamic analyses, the time increment was set equal to $0.002 \mathrm{sec}$, considering that the magnitude of acceleration for initiation of rocking motion is not influenced by the time step size. 
The computed acceleration magnitudes are in good agreement with the load factors obtained using previously presented limit equilibrium formulations (Table 1).

The plot of failure mechanisms for the considered examples is also shown in Figure 3. In the case of dynamic analysis, slight differences can be noted with results obtained from limit equilibrium analysis $[3,14]$ which can be mainly ascribed to the simple model adopted for contact point specification and large displacement regime.

\begin{tabular}{c|c|c|c|c|c|c}
\hline \multirow{2}{*}{ Case study } & $\begin{array}{c}\text { Model size } \\
(b \times c)\end{array}$ & \multicolumn{4}{|c|}{ Point contact formulation } & $\begin{array}{c}\text { Surface contact } \\
\text { formulation }\end{array}$ \\
\cline { 3 - 7 } & & \multicolumn{2}{|c|}{ Limit equilibrium analysis } & \multicolumn{2}{c}{ Dynamic analysis } & $\begin{array}{c}\text { Limit equilibrium } \\
\text { analysis [14] }\end{array}$ \\
\cline { 3 - 7 } & & $\alpha$ & $\begin{array}{c}\text { CPU Time } \\
(\mathrm{s})\end{array}$ & $\mathrm{ag}_{\mathrm{g}}(\mathrm{g})$ & $\begin{array}{c}\text { CPU Time } \\
(\mathrm{s})\end{array}$ & $\alpha$ \\
\hline Example no. 1 & $33 \times 174$ & 0.643 & 0.1 & 0.641 & 80.5 & 0.643 \\
\hline Example no. 2 & $55 \times 290$ & 0.580 & 0.1 & 0.568 & 100.5 & 0.580 \\
\hline Example no. 3 & $46 \times 240$ & 0.404 & 0.1 & 0.387 & 97.5 & 0.404 \\
\hline Example no. 4 & $55 \times 296$ & 0.332 & 0.1 & 0.307 & 103.0 & 0.332 \\
\hline Example no. 5 & $61 \times 300$ & 0.240 & 0.1 & 0.229 & 106.5 & 0.240 \\
\hline Example no. 6 & $146 \times 814$ & 0.348 & 0.2 & 0.302 & 214.0 & 0.348 \\
\hline
\end{tabular}

*Referred to $3.3 \mathrm{GHz} \mathrm{CPU}$, per unit time duration of dynamic analysis (second) in the case of dynamic analysis.

Table 1: In-plane loaded masonry walls: comparison of collapse load factors from LA formulations and accelerations obtained from mathematical programming problems.

In terms of computational efficiency, it is evident that the dynamic formulation is more computationally expensive than limit analysis formulations when the objective of numerical investigations is the determination of failure mechanism and collapse load under constant and uniformly distributed horizontal acceleration. Besides, the adopted dynamic formulation can be conveniently used to investigate masonry panels subjected to earthquake excitation considering that, within the framework of the applications developed so far, a good agreement in terms of structural response and failure mechanism together with numerical stability and computational efficiency were observed. 


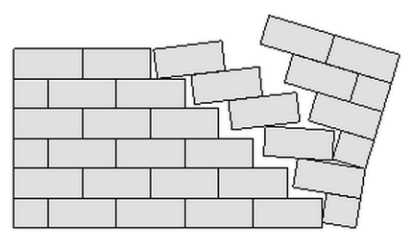

Time $=2.2 \mathrm{~s}$

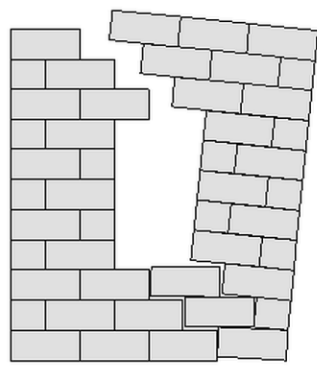

Time $=1.1 \mathrm{~s}$

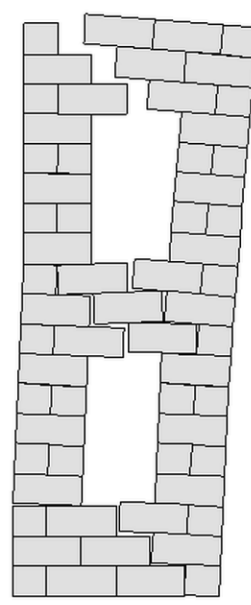

Time $=2.6 \mathrm{~s}$ a)

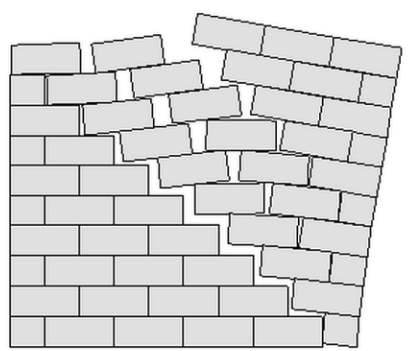

Time $=2.0 \mathrm{~s}$

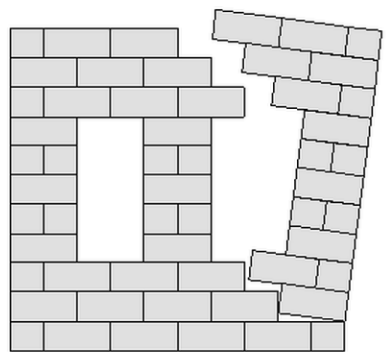

Time $=2.7 \mathrm{~s}$

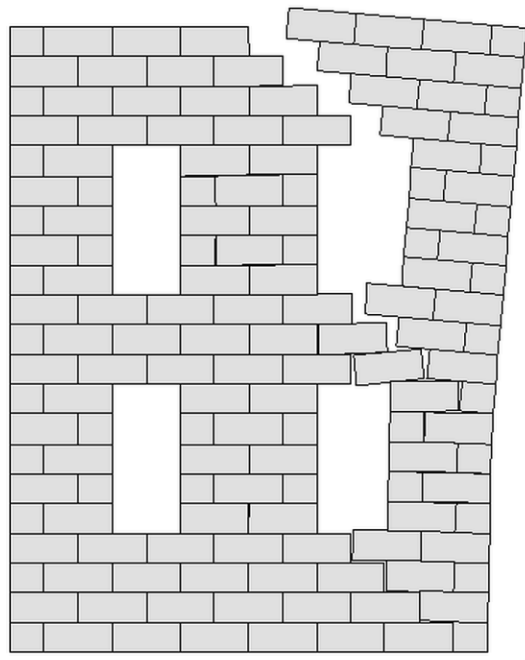

Time $=2.4 \mathrm{~s}$

e) b)

d)

f)

Figure 3: In-plane loaded masonry walls: failure mechanism obtained from the contact dynamic formulation considering a constant and uniformly distributed acceleration value.

\section{CONCLUSIONS}

- Two formulations for limit equilibrium and dynamic analysis of masonry block structures were presented in this paper. The formulations are based on a point contact model, assume a no-tension, associative frictional behavior at interfaces and use mathematical programming to solve the associated contact problems.

- The formulations were applied to a set of dry-jointed masonry block panel from the literature and a good agreement in terms of failure mechanism and collapse load was observed with outcomes from the literature using different (surface) contact models. 


\section{ACKNOWLEDGEMENTS}

The financial support of PRIN 2015 Programme by the Ministry of Education, University and Research (MIUR) is gratefully acknowledged for funding the research project "Protecting the Cultural Heritage from water-soil interaction related threats", which is the main framework of the study presented in this article.

\section{REFERENCES}

[1] R.K. Livesley, A computational model for the limit analysis of three-dimensional masonry structures, Meccanica, 27 (3), pp. 161-172, 1992.

[2] T. Tran-Cao, Collapse analysis of block structures in frictional contact. PhD Thesis. School of civil and environmental engineering. Sydney (Australia): The University of New South Wales, 2009.

[3] F. Portioli, C. Casapulla, M. Gilbert, L. Cascini, Limit analysis of 3D masonry block structures with non-associative frictional joints using cone programming, Computers and Structures, 143, pp. 108-121, 2014.

[4] F. Portioli, C. Casapulla, L. Cascini, An efficient solution procedure for crushing failure in 3D limit analysis of masonry block structures with non-associative frictional joints, International Journal of Solids and Structures, 69-70, pp. 252-266, 2015.

[5] F. Portioli, L. Cascini. Assessment of masonry structures subjected to foundation settlements using rigid block limit analysis, Engineering Structures, 113, pp. 347-361, 2016.

[6] F. Portioli, L. Cascini, M. D'Aniello, R. Landolfo, A rigid block model with cracking units for limit analysis of masonry walls subject to in-plane loads, Civil-Comp Proceedings, 99, 2012.

[7] F. Portioli, L. Cascini, Contact Dynamics of Masonry Block Structures Using Mathematical Programming, Journal of Earthquake Engineering, pp. 1-32, 2016, Article in Press.

[8] K. Krabbenhoft, A.V. Lyamin, J. Huang, M. Vicente da Silva, Granular contact dynamics using mathematical programming methods, Computers and Geotechnics, 43, pp. 165-176, 2012.

[9] K. Krabbenhoft, J. Huang, M.V. Da Silva, A.V. Lyamin, Granular contact dynamics with particle elasticity, Granular Matter, 14 (5), pp. 607-619, 2012.

[10] J. Huang, M.V. da Silva, K. Krabbenhoft, Three-dimensional granular contact dynamics with rolling resistance, Computers and Geotechnics, 49, pp. 289-298, 2013.

[11] J. Meng, J. Huang, D. Sheng, S.W. Sloan, Granular contact dynamics with elastic bond model. Acta Geotech. (2016). doi:10.1007/s11440-016-0481-5.

[12] The MOSEK optimization tools manual (2011). Version 8.0. MOSEK ApS, Denmark 2011. http://www.mosek.com

[13] Ferris, M., Tin-Loi, F. Limit analysis of frictional block assemblies as a mathematical program with complementarity constraints, Int. J. Mech. Sci. 43: 209-224, 2006.

[14] M. Gilbert, C. Casapulla, H.M. Ahmed, Limit analysis of masonry block structures with non-associative frictional joints using linear programming, Computers and Structures, 84 (13-14), pp. 873-887, 2006. 